\title{
Differentiation of lard from other edible fats and oils by means of Fourier transform infrared spectroscopy and chemometrics.
}

\begin{abstract}
Fourier transform infrared (FTIR) spectra at mid infrared regions $(4,000-650 \mathrm{~cm}-1)$ of lard and 16 edible fats and oils were compared and differentiated. The chemometrics of principal component analysis and cluster analysis (CA) was used for such differentiation using FTIR spectra intensities of evaluated fats and oils. With PCA, an "eigenvalue" of about $90 \%$ was achieved using four principal components (PCs) of variables (FTIR spectra absorbances at the selected frequency regions). PC1 accounted for $44.1 \%$ of the variation, while PC2 described $30.2 \%$ of the variation. The main frequency regions that influence the separation of lard from other evaluated fats and oils based on PC1 are 2,852.8 followed by 2,922 and $1,464.7 \mathrm{~cm}-1$. Furthermore, CA can classify lard into its group based on Euclidean distance
\end{abstract}

Keyword: Chemometrics; Differentiation; Fats and oils; FTIR spectroscopy; Lard. 